\author{
International Journal of Innovative Research in \\ Electrical, Electronics, Instrumentation and Control Engineering
}

Vol. 9, Issue 11, November 2021

DOI: $10.17148 /$ IJIREEICE.2021.91103

\title{
Unmanned Aircraft System (UAS) Based Large Area Identification of Basal Stem Rot (BSR) Infected Oil Palm Tree Using Convolutional Neural Network (CNN)
}

\author{
K. Johnathan ${ }^{1}$, T.S.Y. Moh ${ }^{2, *}$ \\ Undergraduate Student, Department of Electrical and Electronics, School of Engineering \& Technology 1 \\ Assoc. Prof., Department of Electrical and Electronics, School of Engineering \& Technology 2* \\ University College of Technology Sarawak, Sibu, Sarawak, Malaysia.
}

\begin{abstract}
Malaysia is the second largest palm oil producer in the world but ironically, the overall production of the palm oil is declining versus planned growth trajectory. One of the factors affecting the exponential growth of the palm oil estate is fungal or bacterial infection. One of the major diseases that infected palm oil tree is Ganoderma Boninense that which known as Basal Stem Rot (BSR). A fast, accurate, wide-coverage and non-destructive method for detection of infected oil palm tree is required. Therefore, low-cost unmanned aircraft for detection of the health status of palm tree plantation was implemented and image processing of the data collected from the low-cost unmanned aircraft system was processed for oil palm tree health status. MATLAB based detection was implemented. Convolutional Neural Network (CNN) and few image processing techniques were introduced in this project.
\end{abstract}

Keywords: Basal Stem Rot (BSR), Unmanned Aircraft System (UAS), Convolutional Neural Network (CNN), detection

\section{INTRODUCTION}

Nowadays, derivatives of palm oil have been used in a lot of different products and industries globally. Production and consumption of world's oil and fats increased at almost the same rates as in the past. Malaysia is the second largest palm oil producer in the world [1-3]. Oil palm has the highest average productivity compared to other major crops. Based on the data collected by Malaysian Palm Oil Board, Sarawak produced 5.7 million tonnes of crude palm oil, 4.8 million tonnes of crude palm oil, 5.2 million tonnes of crude palm oil and 5.1 million tonnes of crude palm oil in 2015, 2016, 2017 and 2018 respectively [4]. Up to date, with more trees maturing, state is expected to achieve 6 million tonnes and beyond. Throughout the analysis from Malaysian Palm Oil Board, the production of the palm oil is decreased [4]. The factors that caused the decrease of production oil palm are the environment, labour shortage, land availability, pricing and destructive disease on the oil palm. One of the main concerns is fungal or bacterial infection [5]. Once affected, it may wipe out the entire plantation in a very fast speed. Therefore, early detection is very important to reduce the loss and it was reported that this alone was costing some South East Asian countries around US\$500 million a year [6]. It was reported that the industry is facing a destructive crop disease which is Ganoderma disease or commonly known as Basal Stem Rot (BSR) that mainly caused by a fungal called Ganoderma boninense [6, 7]. Basal Stem Rot (BSR) is the main pathogen that impact the oil palm trees and kill it which affected the production of the oil palm. It was recorded on an average of $50 \%$ production losses from $80 \%$ of 12 -year-old oil palm trees in coastal area of Malaysia [7]. Currently, although it is impossible to manage a field free of this dreadful disease, considerable reduction of the disease can be achieved through a proper management system [6]. Since that by visual inspection and monitoring of the whole palm estate would be quite a challenge due to accessibility difficulty, drawn-out and high-priced at the early stages and the current known effective way to stop disease from spreading to other oil palm trees is by removing the infected parts of oil palm trees at which we known this as the surgery of oil palm tree, therefore it is very important to have a fast, accurate, large area and harmless method for detection of infected oil palm tree. In this project, a low-cost UAS-based drone had been used as the supportive tool to collect the training data images. Images had been collected and will be trained by using CNN algorithm which enable the network to classify the infected tree. Relevant detection algorithm will be developed using MATLAB software which able to detect the BSR oil palm tree through software.

\section{LITERATURE REVIEW}

Previous studies done by Ortiz et. al demonstrated the possibility of applying similar approach to oil palm health detection which are weed mapping in sunflower crops using unmanned aerial vehicles and a crop row detection method [8,9]. In this study, UAS had been used to map weed and the model been used is quadcopter platform with vertical take-off and landing, model md4-1000. This study consists of 49 square white frames and every frame was labelled with a GPS 


\section{IJIREEICE}

\section{International Journal of Innovative Research in Electrical, Electronics, Instrumentation and Control Engineering}

Vol. 9, Issue 11, November 2021

\section{DOI: 10.17148/IJIREEICE.2021.91103}

coordinate and captured for comparison of on-ground weed infestation and outputs from image classification. This studies validated some of the main hypothesis in the literature concerning the flight height and the sensor to use. Luis et. al demonstrated the site detection by using UAS system and geometry-based image segmentation [10]. The author had used three datasets and each dataset was obtained using three different systems. The author had evaluated two image segmentation technique which are k-means clustering and graph-cut based segmentation and both segmentations showing similar performance. In this experiment, the author had achieved detection rate of 97.76 and achieving average performance of $93 \%$ frames using proper detection. A deep learning object detection and traditional object tracking by using UAS had been studied by Koskowich et. al [11]. The author had designed an application of monoplotting enabling monitoring of the results for deep learning object detection and traditional object detection [11]. The dataset to be analysed from fixed-wing UAS and tested through four types of tracking algorithm. The method that had been used by author is firstly define region of interest, define keypoint and then detect in regions of interest, compute homography and compute occlusion mask and next apply tracking to detections and lastly transform tracked coordinates via homography to aerial image. The detection and tracking accuracy are $99.99 \%$ at object detection which the outputs were passed to the tracking algorithm evaluated. From all the previous work that were reviewed and published, UAS systems are found to be suitable to apply in this project[12]. In this project, UAS can be used as the supporting tool for training data collection.

Basal Stem Rot have few major distinctive visual differences as outlined by Elliott et. al who observed that infected oil palm tree has symptoms such as butt rot that includes a mild to severe wilt, reduced growth, overall off -colour foliage and older fronds are eventually drop [13]. Khairuniza et. al also studied about the symptoms of Basal Stem Root disease [14]. The author stated that the symptoms of infected oil palms are few unopened new fronds, green fronds wilts and hanging downward like a skirt, fronds become yellowish, small canopy due to smaller fronds production and existence of Basidiomata of the pathogen on the trunks. These distinctive symptoms of the disease could be visually observed and grouped as (1) mature leaves wilt (yielding yellow colour visually) and (2) trunk falling due malnutrition [3, 13]. This disease caused the disrupting distribution of water and nutrients in the tree and subseqeuntly reduced of productivity of the oil palm up to $80 \%$ every years [15]. Current industry practise is to remove oil palm tree that was infected with more than $25 \%$ with Ganoderma disease.

Study done by Dimitris et al demonstrated the suitability, relevancy and comparison of UAS image processing techniques with this project [16]. In this review, author had used two types of image processing software which are Agisoft Photoscan and Imagine UAS. The author stated Imagine UAS has bigger advantages because it provides the ability to measure area, volume, and create cross sections. In addition, these two software tools are almost equivalent, while the accuracies of their products are similar to the accuracies of the external orientations of drone images. As for image segmentation, $\mathrm{k}-$ means clustering was widely used to categorize the pixels from the image into k-clusters with each cluster having a limitation which classifies pixels into a single cluster [17-19]. Dhanachandra et. al demonstrated a success in image segmentation using K-mean clustering algorithm and subtractive clustering algorithm [20]. The author had segmented an image by using k- mean clustering algorithm and through subtractive cluster to generate the output image. The subtractive cluster had also tune by varying the hyper sphere cluster radius. In addition, Nurhayati et. al. successfully demonstrated the detection of the beef quality using mobile-based K-Mean clustering method in pursue of comparison and classification of segmentation and feature extraction the meat quality and monitoring [21]. The author concluded that the result of the detection on the meat quality from the application showed the percentage of the good level of achievement and the level of achievement by using k-mean clustering method was at $80 \%$. The study of improved gaussian mixture model with expectation - maximiziation for clustering of remote sensing imagery had been done by Neagoe et. al [22]. In this study, author had reviewed the performance of $\mathrm{k}$ - mean clustering method and Gaussian Mixture Model with expectation maximization clustering method. Another study had been done by Shukui et. al on the image clustering which used mean shift algorithm [23]. The author reported on the mean shift clustering method compared with other general clustering on the same experimental image to evaluate and compare the classification accuracy. Singh et al commented about the performance evaluation of enhanced hierarchical and partitioning based clustering algorithm [24].

Image classification techniques are used to extract on desired feature/information based on the segmented images and classify based on the region of interest that user required. Algorithm or detection techniques for image classification are different and unique compared to image segmentation. Feature extraction algorithm was first demonstrated by Pinto et. al [17]. The author used the feature extraction algorithm to classify the crop and plant disease. The team also stated that the accuracy can be improved by using various other image enhancement techniques and by increasing the size of the training dataset. 4 machine learning classifiers had been investigated in this paper which are K-Nearest Neighbors (KNN), Native Bayes Classifier, Support Vector Machine (SVM) and Multinomial Logistic Regression. On the other hand, Convolutional neural networks (CNNs) are widely used in pattern- and image -recognition problems as they have a number of advantages compared to other techniques. A study of plant classification using convolutional neural network 


\section{IJIREEICE}

\section{International Journal of Innovative Research in \\ Electrical, Electronics, Instrumentation and Control Engineering}

Vol. 9, Issue 11, November 2021

\section{DOI: $10.17148 /$ IJIREEICE.2021.91103}

was demonstrated by Yalcin et. al [25]. The author had showed the structure of their CNN model and the overall framework and layers of approaches. The experiment result showed that CNN based approach obtained an accuracy of 97.47\% on 16 different kinds of plants and other classifiers had lower accuracy than CNN based approach which lowest is $74.92 \%$. Related study on the Deep Learning based oil palm tree detection and counting for high-resolution remote sensing images had been demonstrated by Weijia et. al [26]. The author had proposed a deep learning-based framework for oil palm tree detection which able to count oil palm tree by using high-resolution remote sensing images for Malaysia. In addition, author also compared the CNN techniques with other three tree detection methods such as Artificial Neural Network (ANN), template matching and local maximum filter in order to get the result of accuracy. Through comparing the accuracy of this method with other three method, CNN method reported to have higher accuracies ranging from $96 \%$ to $99 \%$ relatively[26]. In parallel, counting crop plant by deep learning was first demonstrated by Javier et. al which using Convolutional Neural Network (CNN) [27]. In this study, the author noticed that when the plants were clustered, the label miscount occurred and to address these problems, author had employ the Mean Square Error as a common loss function to be minimized [27].

Work related to Support Vector Machine algorithm was primarily demonstrated by Ahmad et. al which used image processing on palm oil leaf disease detection by using K-mean clustering and Multiclass SVM (Support Vector Machine) classifier [28]. Author had planned the methodology on image processing which start with image acquisition, contrast enhancement, conversion of RGB image to various colour spaces, K-mean clustering, feature extraction and lastly Multiclass SVM classifier. Kernel based learning approach for satellite image classification using support vector machine was carried out by Moorthi et. al [29]. The experiment had been done by using the dataset that had been collected by National Remote Sensing Agency (NRSC) and used two classification methods which are Maximum likelihood (MXL) and Support vector machine (SVM). Padol et. al studied on fusion classification technique used to detect downy and powdery mildew grape leaf diseases [30]. In this study, the author had done the classification by applying Support vector machine (SVM) and artificial neural network (ANN) individually and proposed a fusion classification technique that from SVM and ANN for grape leaf disease detection. Throughout the literature review and accuracy evaluation of all the related algorithms, CNN had reported to the highest accuracy compared to others in image classification and K-mean clustering algorithm were selected to be used.

\section{METHODOLOGY}

The programming workflow is split into several parts, which were training of network, classification and detection as illustrated in Figure 1 respectively. Parameter optimisation was carried out by five different situations of training set. After training, the CNN model was used for classification and detection. For classification and detection, the created testing image will go through image enhancement to enhance the feature, image segmentation for segmentation of the region of interest (ROI) and bound box method for pin point the ROI. In bound box method, each box will be cropped and go through the image classification in the detection by looping function. After the image classification, label prediction was marked on the image and showing the number of the infected tree detected. The results were stored for cross checking. On the ground training data based on a local company oil plantation estate was collected using UAS (model DJI Spark May 2017) which has specification of a camera of 12MP, battery capacity of $1480 \mathrm{mAh}$, max transmission distance 100m (Distance), 50m (Height) and Vertical Take Off and Landing. The height that the device for capturing all images is set to be at constant of $40 \mathrm{~m}$ high when capturing all training data sets. This is due to the device's maximum allowable operation height. Images captured were saved locally and to be used as training data set. A total of 500 images were captured with 200 of healthy tree images and 300 of infected tree images being prepared for training data images. Example of images used healthy tree and infected tree training are shown in Figure 1 respectively.

MATLAB platform was used for further coding, imagine segmentation, extraction, training, testing and validation of this project. Image acquisition is very important as the quality of the image plays an important role in the image processing and image classification. After inserting the image into workspace, the step of pre-processing the image will be done to enable analysis. For the pre-process, image enhancement is done by using the correlation stretch algorithm which enhance the image to red, green and blue based on the hue. After the correlation stretch had done, $\mathrm{k}-$ mean clustering was used to segment the image and also the feature extraction. For the k-mean clustering, the input image will be converted into colour map which provides information on luminosity and relevant colours ranging from blue to yellow. Euclidean distance $d$ had been used to compute each pixel of image and assign all the pixels to the nearest centre based on distance. After the clustering, feature extraction will be done for result analysis purpose. On feature extraction part, image was converted into binary image and went through subtraction method to keep the green colour map for the detection of oil palm tree. Bound box method was used in this project to create outline of the oil palm tree. After the object detection had been done, the image for the region of interest will be cropped by using imcrop function for image classification purpose. Image classification will go on by using pre-trained network which is Convolutional neural network (CNN) to classify 


\section{International Journal of Innovative Research in \\ Electrical, Electronics, Instrumentation and Control Engineering}

Vol. 9, Issue 11, November 2021

\section{DOI: $10.17148 /$ IJIREEICE.2021.91103}

the healthy and infected oil palm tree. When the classifier had classified the oil palm tree, the infected oil palm tree will be pin pointed and create outline on the infected oil palm to acknowledge the user location of the infected oil palm tree. After the image being processed and filtered, total amount of the infected oil palm tree was to be shown accordingly.
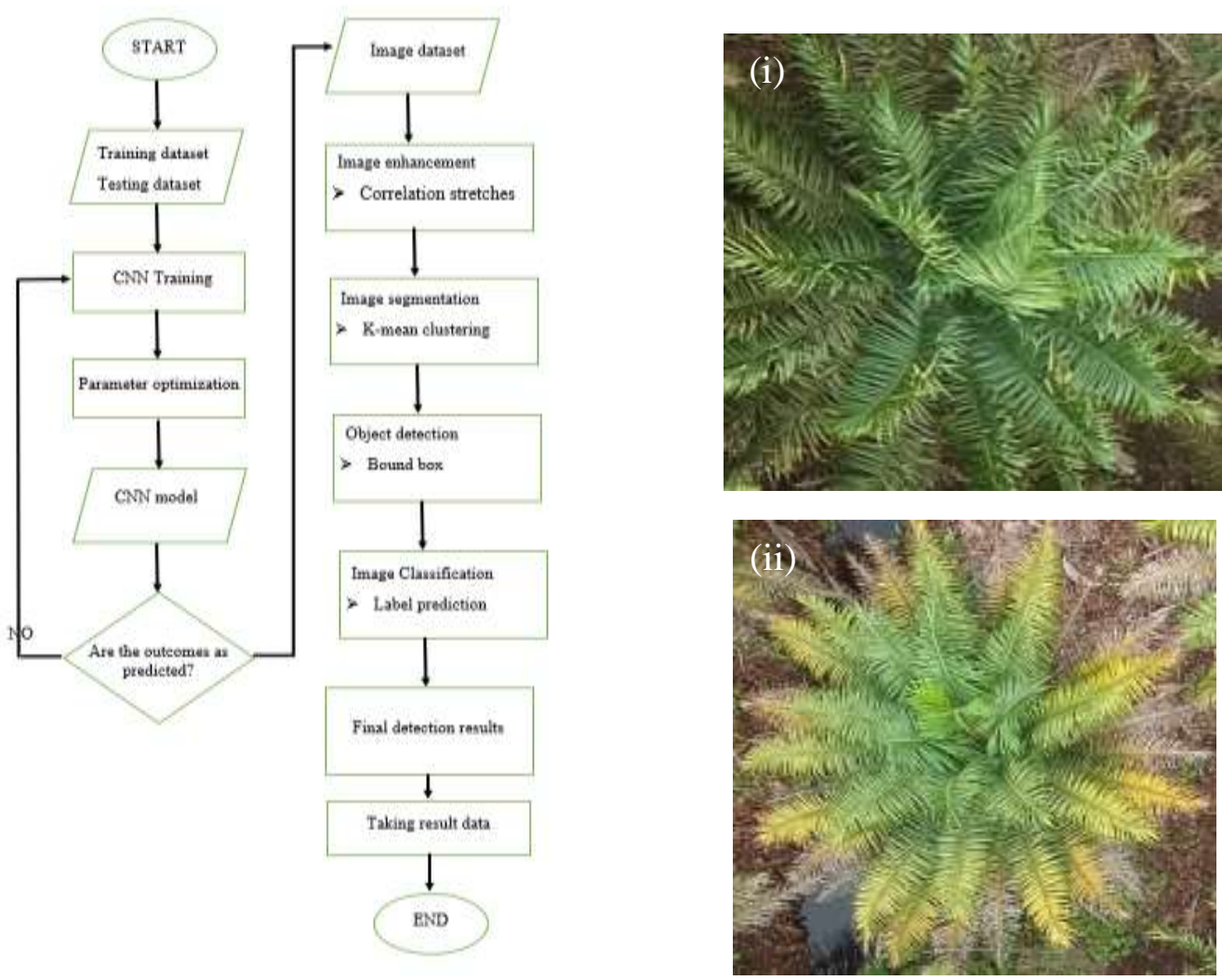

Figure 1: Large scale detection and counting of oil palm infected by Ganoderma Boninense fungal with corresponding image (a) Overall flow chart and (b) Example of training data image captured by UAS at 40 m height with (i) Healthy oil plam tree versus (ii) Infected oil palm tree.

Convolutional neural network from MATLAB Deep Learning toolbox was selected and used in this project. 500 images of data set which has scattering of 200 of healthy tree images and 300 of infected tree images with each image size of $400 \times 400$ pixels are being used for this model training purpose. The training process will go through 5 convolutional layers, 5 max pooling layers and one fully connected layer. The training option for the network is 128 of Minibatchsize, 100 MaxEpochs which 2 iteration for each Epoch and the training will be fully utilise the CPU of the computer. A graphic user interface (GUI) was created for the ease of user interface and handling.

\section{RESULT AND DISCUSSION}

A total of 500 on-the ground oil palm plantation images were captured accordingly based on the requirements as per above for training network purposes. Out of 500 images, $60 \%$ of the images were classified as infected tree and remaining $40 \%$ of the images as healthy tree. From $60 \%$ or 300 images, 25 images were labelled as $100 \%$ infected tree, 50 images for $75 \%$ infected tree, 120 images for $50 \%$ infected tree, 105 images for $25 \%$ infected tree. Figure 2 shows randomly selected sample of images used for training. Minimum requirement for the image is 500*500 which low size of image may cause low accuracy of the training. The CNN model was trained with 600 iteration in order for the network to learn the feature of the infected tree and the healthy tree based on the training set images. CNN model is designed with various convolutional layers such as Rectified Linear Unit (ReLU) layers, max pooling layers, fully connected layer and classification layer. During the training of the network, the training process will pop out in window for user to monitor the process of training as showed in Figure 2(f) and Figure 2(g) summarized on the respective training accuracy for 5 convolution layers. The training condition with 2 convolutional layers had the highest accuracy of $95.5 \%$ and the more convolutional layers in the training process, the training accuracy getting lower mainly due to overfitting of the network. 
International Journal of Innovative Research in Electrical, Electronics, Instrumentation and Control Engineering

Vol. 9, Issue 11, November 2021

\section{DOI: $10.17148 /$ IJIREEICE.2021.91103}
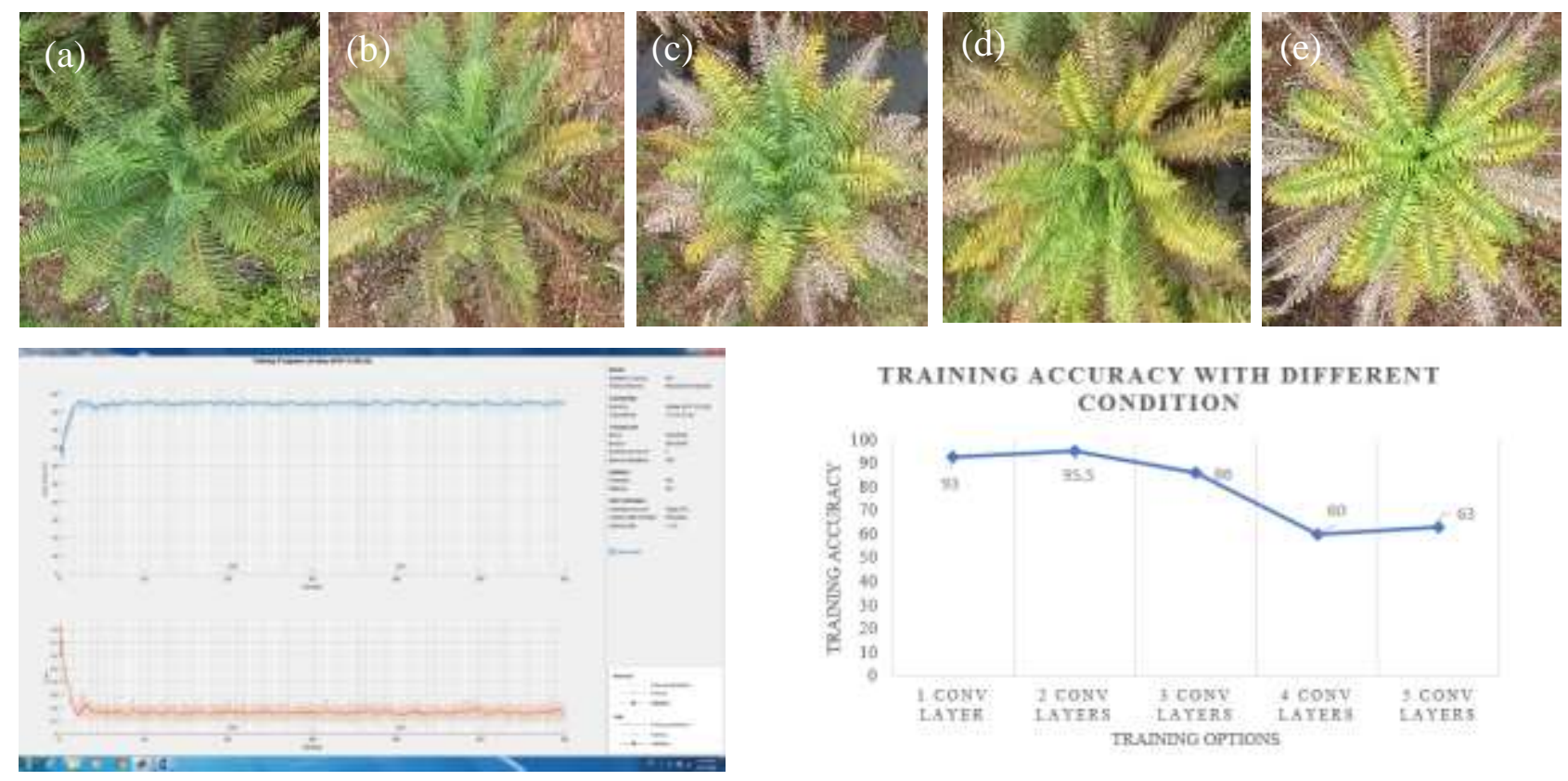

Figure 2: Randomly selected sample of images used for network training with percentage of classified infection ranging from (a) $0 \%$, (b) $25 \%$, (c) $50 \%$, (d) $75 \%$ and (e) $100 \%$. Image (f) shows the partial monitoring of training related to 2 convolutional pooling layers from designed GUI and (g) illustrates the training accuracy comparisons for respective 5 convolution layers.

Table 1: Detection results for each of the corresponding level of infected trees with respective level of infected tree starting with (a) $25 \%$ of infection, (b) $50 \%$ of infection, (c) $75 \%$ of infection and lastly (d) $100 \%$ of infection.

\begin{tabular}{|c|c|c|c|c|c|c|c|}
\hline (a) 25\% Infected Trees & \multicolumn{5}{|c|}{ Total Successful Detected Number Of } & \multirow{2}{*}{$\begin{array}{c}\text { Average Of Correctly Detected } \\
\text { Number Of Trees (\%) }\end{array}$} \\
\hline Target (Number Of Trees) & $1^{\text {st }}$ & 2nd & 3rd & 4th & 5 th & 80.0 \\
\hline $1 / 25$ & 1 & 0 & 1 & 1 & 1 & 36.0 \\
\hline $5 / 25$ & 2 & 1 & 2 & 2 & 2 & 52.0 \\
\hline $10 / 25$ & 5 & 6 & 5 & 5 & 5 & 69.3 \\
\hline $15 / 25$ & 11 & 10 & 10 & 10 & 11 & 64.0 \\
\hline $20 / 25$ & 13 & 13 & 13 & 12 & 13 & 59.2 \\
\hline $25 / 25$ & 14 & 15 & 16 & 15 & 14 & \\
\hline
\end{tabular}

\begin{tabular}{|c|c|c|c|c|c|c|}
\hline (b) 50\% Infected Trees & \multicolumn{6}{|c|}{ Total Successful Detected Number Of } \\
Trees For Each Cycle & \multirow{2}{*}{$\begin{array}{c}\text { Average Of Correctly Detected } \\
\text { Number Of Trees (\%) }\end{array}$} \\
\hline Target (Number Of Trees) & 1 st & 2nd & 3rd & 4 th & 5th & 100.0 \\
\hline $1 / 25$ & 1 & 1 & 1 & 1 & 1 & 56.0 \\
\hline $5 / 25$ & 2 & 3 & 2 & 3 & 4 & 54.0 \\
\hline $10 / 25$ & 5 & 5 & 6 & 6 & 5 & 70.7 \\
\hline $15 / 25$ & 10 & 10 & 10 & 11 & 12 & 71.0 \\
\hline $20 / 25$ & 14 & 14 & 15 & 15 & 13 & 96.8 \\
\hline $25 / 25$ & 24 & 24 & 24 & 25 & 24 & \\
\hline
\end{tabular}


International Journal of Innovative Research in Electrical, Electronics, Instrumentation and Control Engineering

Vol. 9, Issue 11, November 2021

DOI: $10.17148 / I J I R E E I C E .2021 .91103$

\begin{tabular}{|c|c|c|c|c|c|c|}
\hline \multirow{2}{*}{$\begin{array}{l}\text { (c) } 75 \% \text { Infected Trees } \\
\text { Target (Number Of Trees) }\end{array}$} & \multicolumn{5}{|c|}{$\begin{array}{l}\text { Total Successful Detected Number Of } \\
\text { Trees For Each Cycle }\end{array}$} & \multirow{2}{*}{$\begin{array}{l}\text { Average Of Correctly Detected } \\
\text { Number Of Trees }(\%)\end{array}$} \\
\hline & $1 \mathrm{st}$ & 2nd & 3rd & 4th & 5 th & \\
\hline $1 / 25$ & 1 & 1 & 1 & 1 & 1 & 100 \\
\hline $5 / 25$ & 3 & 3 & 4 & 3 & 4 & 68 \\
\hline $10 / 25$ & 8 & 9 & 8 & 9 & 9 & 86 \\
\hline $15 / 25$ & 10 & 11 & 11 & 10 & 12 & 72 \\
\hline $20 / 25$ & 15 & 15 & 16 & 16 & 15 & 77 \\
\hline $25 / 25$ & 25 & 24 & 24 & 25 & 25 & 98.4 \\
\hline
\end{tabular}

\begin{tabular}{|c|c|c|c|c|c|c|}
\hline \multirow{2}{*}{$\begin{array}{l}\text { (d) } 100 \% \text { Infected Trees } \\
\text { Target (Number Of Trees) }\end{array}$} & \multicolumn{5}{|c|}{$\begin{array}{c}\text { Total Successful Detected Number Of } \\
\text { Trees For Each Cycle }\end{array}$} & \multirow{2}{*}{$\begin{array}{l}\text { Average Of Correctly Detected } \\
\text { Number Of Trees (\%) }\end{array}$} \\
\hline & $1 \mathrm{st}$ & 2nd & $3 \mathrm{rd}$ & 4 th & 5 th & \\
\hline $1 / 25$ & 1 & 1 & 1 & 0 & 1 & 80.0 \\
\hline $5 / 25$ & 2 & 4 & 3 & 4 & 5 & 72.0 \\
\hline $10 / 25$ & 6 & 6 & 7 & 7 & 7 & 66.0 \\
\hline $15 / 25$ & 10 & 11 & 11 & 12 & 12 & 74.7 \\
\hline $20 / 25$ & 17 & 18 & 18 & 17 & 19 & 89.0 \\
\hline $25 / 25$ & 25 & 24 & 24 & 25 & 24 & 97.6 \\
\hline
\end{tabular}

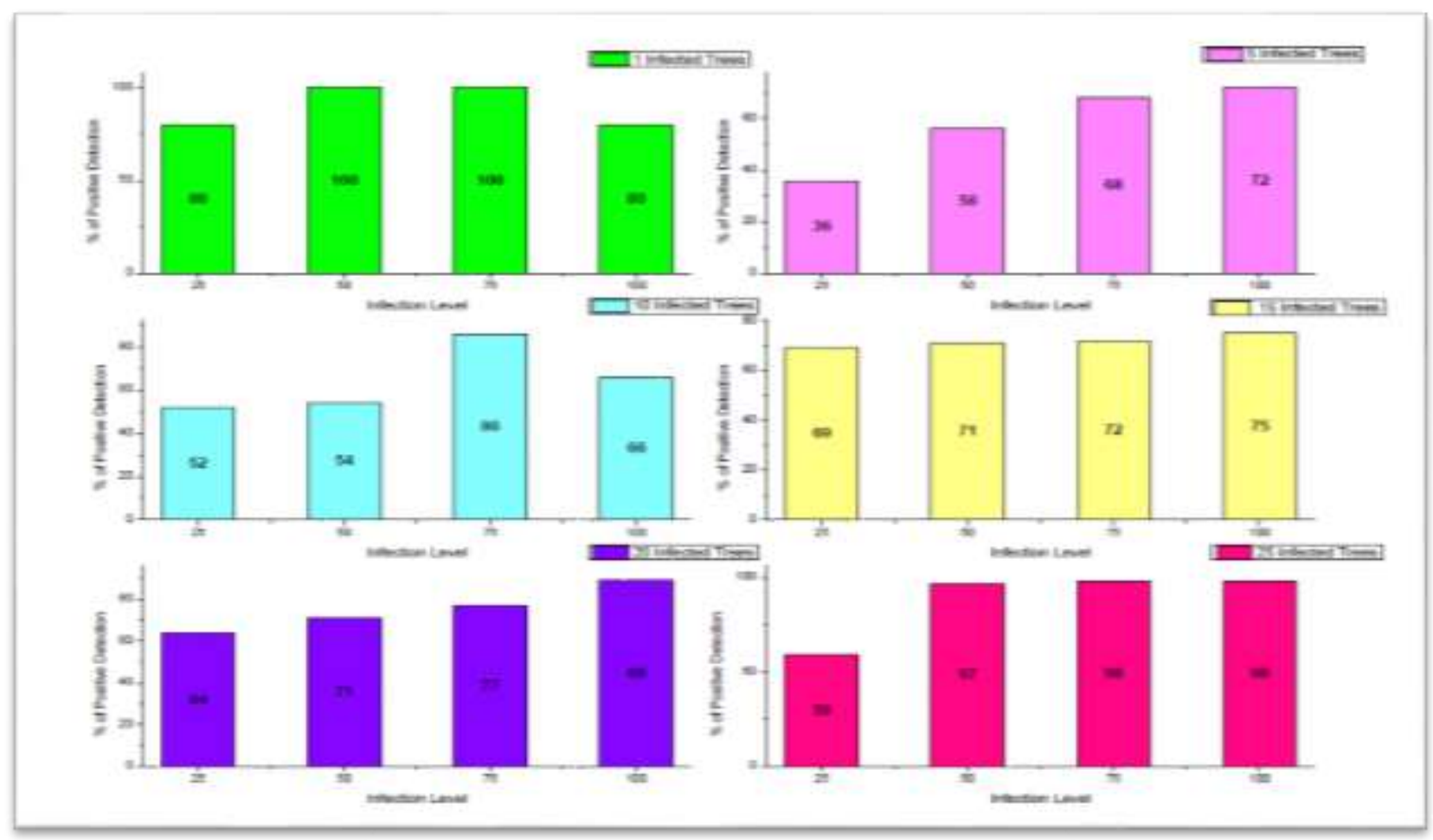

Figure 2: Results summary showing corresponding percentage (\%) of positive detection of infected tree versus infection level for each known number of infected trees respectively ranging from 1-25 infected trees

In order to test and validate the accuracy and success of CNN network training, derivative images were used as raw testing input images. A total of 720 images ( 6 target x 6 images x 5 testing cycles $\mathrm{x} 4$ cases) were involved in the testing and validation of the trained network. These 4 groups are referring to the input images that contained minimum $25 \%$ of the image are make-up with infected tree up to $100 \%$ with incremental value of $25 \%$ of infected tree for each cycle. The 


\section{International Journal of Innovative Research in \\ Electrical, Electronics, Instrumentation and Control Engineering}

Vol. 9, Issue 11, November 2021

\section{DOI: $10.17148 /$ IJIREEICE.2021.91103}

exact location and coordinate of the known infected trees were placed in randomly manner and in location-unique manner (no overlapping in term of location). Testing condition and corresponding results for each case are summarized in Table 1 respectively.

\section{CONCLUSION}

From this project, several objectives have been achieved. The suitability of using low cost UAS system to detect/ monitor health status of an oil palm tree had been studied extensively. From all the previous work that been reviewed, UAS had been used in many industries and many purposes. Mainly, it starts with military and now it develops to other industries such as geomorphology and etc. UAS system is suitable and relevant to be applied in detection/monitoring of large scale oil palm tree health status. Besides, the detection techniques to detect early sign of Ganoderma Boninense had been successfully carried out by using Convolutional Neural Network and few image processing techniques. In CNN, a set of pre-labelled images need to be trained on the network to enable the network to classify the infected oil palm tree. 500 datasets for the training had been collected by using DJI Spark from the height of 40m. The network had been trained in five condition which is 1 convolutional pooling layer, 2 convolutional pooling layers until 5 convolutional pooling layers. The trained network will go through matching testing and actual detection testing for the matching accuracy. The training accuracy for 2 convolutional pooling layers had the highest accuracy of 95.5\%. The k-mean clustering had been carried out successfully which image processing had been done. Lastly, this study reported the success of positive detection of BSR affected tree for case $1-25 \%$ (accuracy of 36\%-80\%), case $2-50 \%$ (accuracy of 54\%-100\%), case $3-75 \%$ (accuracy of 68\% - 100\%) and case $4-100 \%$ (accuracy of 66\% - 97.6\%). It could be observed that the higher the infection rate, the detection accuracy will be much higher and vice-versa.

\section{VI.ACKNOWLEDGEMENT}

The authors would like to thank maintenance staff for their support in access to the lab access beyond working hours and facilities supports.

\section{REFERENCES}

[1] H. Varkkey, "The Growth and Prospects for the Oil Palm Plantation Industry in Indonesia," Oil Palm Industry Economic Journal, Journal vol. 12(2), September 20122012.

[2] Nickson E. Otieno1, Xeuping Dai2, Daniele De Barba2, Abbassi Bahman2, Elise Smedbol2,Marouan Rajeb2, Lise Jaton2, "Palm Oil Production in Malaysia: An Analytical Systems Model for Balancing Economic Prosperity, Forest Conservation and Social Welfare," Agricultural Sciences, vol. 7, pp. 55-69, 17 February 20162016.

[3] W. Verheye, "Growth and Production of Oil Palm," p. 25, 2010. Land Use, Land Cover and Soil Sciences. Encyclopedia of Life Support Systems (EOLSS), UNESCO-EOLSS Publishers, Oxford, UK.

[4] M. P. O. Board, "PRODUCTION OF CRUDE PALM OIL FOR THE MONTH OF DECEMBER 2016."

[5] T. C. Hai, "Land Use and The Oil Palm Industry in Malaysia," November 20002000.

[6] R. Hushiarian, N. A. Yusof, and S. W. Dutse, "Detection and control of Ganoderma boninense: strategies and perspectives," SpringerPlus, vol. 2 , no. 1 , p. $555,2013 / / 2013$.

[7] AW Nurul Isma1, AS Idris3, N Rosimah1, O Hishamuddin2, A Mohd. Puad2, "Capability of Bioorganic Fertilizer Pseudomonas GanoEB3 for suppressing Basal Stem Rot Disease in Oil Palm Seedlings," vol. 1, no. 5, 2015.

[8] M. Pérez-Ortiz, J. M. Peña, P. A. Gutiérrez, J. Torres-Sánchez, C. Hervás-Martínez, and F. López-Granados, "A semi-supervised system for weed mapping in sunflower crops using unmanned aerial vehicles and a crop row detection method," Applied Soft Computing, vol. 37, pp. 533-544, $12 / / 2015$.

[9] Johnathan K, Moh TSY, and International Journal of Advanced Research in Electrical, "Review on Low-Cost Unmanned Aircraft System (UAS) and Digital Image Processing Techniques for Large Scale Oil Palm Tree Health Detection Application," vol. 9, no. 10, pp. 2651 - $2658,2020$.

[10] L. Mejias and D. Fitzgerald, A multi-layered approach for site detection in UAS emergency landing scenarios using geometry-based image segmentation.

[11] B. J. Koskowich, M. Rahnemoonfai, and M. Starek, "Virtualot - A Framework Enabling Real-Time Coordinate Transformation \&amp; Occlusion Sensitive Tracking Using UAS Products, Deep Learning Object Detection \&amp; Traditional Object Tracking Techniques," in IGARSS 2018 - 2018 IEEE International Geoscience and Remote Sensing Symposium, 2018, pp. 6416-6419.

[12] K. Johnathan; T.S.Y. Moh, "Review on Low-Cost Unmanned Aircraft System (UAS) and Digital Image Processing Techniques for Large Scale Oil Palm Tree Health Detection Application," International Journal of Advanced Research in Electrical, Electronics and Instrumentation Engineering, vol. 9, no. 10, pp. 2651 - 2658, 2020.

[13] J. Y. U. M. L. Elliott, "Diseases and Disorders of Ornamental Palms " March 20042004.

[14] S. Bejo and C. N. Vong, Detection of Basal Stem Rot (BSR) Infected Oil Palm Tree Using Laser Scanning Data. 2014, pp. 156-164.

[15] M. A. Markom et al., "Intelligent electronic nose system for basal stem rot disease detection," Computers and Electronics in Agriculture, vol. 66, no. 2, pp. 140-146, 5// 2009.

[16] K. Dimitris, P. Petros, and S. Maria, "UAV and the comparison of image processing software," International Journal of Intelligent Unmanned Systems, vol. 5, no. 1, pp. 18-27, 2017/01/03 2017.

[17] L. S. Pinto, A. Ray, M. U. Reddy, P. Perumal, and P. Aishwarya, "Crop disease classification using texture analysis," in 2016 IEEE International Conference on Recent Trends in Electronics, Information \& Communication Technology (RTEICT), 2016, pp. 825-828.

[18] R. M. Prakash, G. P. Saraswathy, G. Ramalakshmi, K. H. Mangaleswari, and T. Kaviya, "Detection of leaf diseases and classification using digital image processing," in 2017 International Conference on Innovations in Information, Embedded and Communication Systems (ICIIECS), 2017, pp. 1-4. 


\section{International Journal of Innovative Research in Electrical, Electronics, Instrumentation and Control Engineering}

Vol. 9, Issue 11, November 2021

\section{DOI: $10.17148 / I J I R E E I C E .2021 .91103$}

[19] Y. A. Wicaksono, A. Rizaldy, S. Fahriah, and M. A. Soeleman, "Improve image segmentation based on closed form matting using K-means clustering," in 2017 International Seminar on Application for Technology of Information and Communication (iSemantic), 2017, pp. 26-30.

[20] N. Dhanachandra, K. Manglem, and Y. J. Chanu, "Image Segmentation Using K -means Clustering Algorithm and Subtractive Clustering Algorithm," Procedia Computer Science, vol. 54, pp. 764-771, 2015/01/01/ 2015.

[21] O. D. Nurhayati, K. Adi, and S. Pujiyanto, "Detection of the beef quality: Using mobile-based K-mean clustering method," in $20163 r d$ International Conference on Information Technology, Computer, and Electrical Engineering (ICITACEE), 2016, pp. $253-259$.

[22] V. Neagoe and V. Chirila-Berbentea, "Improved Gaussian mixture model with expectation-maximization for clustering of remote sensing imagery," in 2016 IEEE International Geoscience and Remote Sensing Symposium (IGARSS), 2016, pp. 3063-3065.

[23] S. Bo and Y. Jing, "Image Clustering Using Mean Shift Algorithm," in 2012 Fourth International Conference on Computational Intelligence and Communication Networks, 2012, pp. 327-330.

[24] G. Singh, J. Kaur, and Y. Mulge, "Performance evaluation of enhanced hierarchical and partitioning based clustering algorithm (EPBCA) in data mining," in 2015 International Conference on Applied and Theoretical Computing and Communication Technology (iCATccT), 2015, pp. 805810 .

[25] H. Yalcin and S. Razavi, "Plant classification using convolutional neural networks," in 2016 Fifth International Conference on AgroGeoinformatics (Agro-Geoinformatics), 2016, pp. 1-5.

[26] W. Li, H. Fu, L. Yu, and A. Cracknell, "Deep Learning Based Oil Palm Tree Detection and Counting for High-Resolution Remote Sensing Images," Remote Sensing, vol. 9, no. 1, 2017.

[27] J. Ribera, Y. Chen, C. Boomsma, and E. J. Delp, "Counting plants using deep learning," in 2017 IEEE Global Conference on Signal and Information Processing (GlobalSIP), 2017, pp. 1344-1348.

[28] A. N. I. Masazhar and M. M. Kamal, "Digital image processing technique for palm oil leaf disease detection using multiclass SVM classifier," in 2017 IEEE 4th International Conference on Smart Instrumentation, Measurement and Application (ICSIMA), 2017, pp. 1-6.

[29] S. M. Moorthi, I. Misra, R. Kaur, N. P. Darji, and R. Ramakrishnan, "Kernel based learning approach for satellite image classification using support vector machine," in 2011 IEEE Recent Advances in Intelligent Computational Systems, 2011, pp. 107-110.

[30] P. B. Padol and S. D. Sawant, "Fusion classification technique used to detect downy and Powdery Mildew grape leaf diseases," in 2016 International Conference on Global Trends in Signal Processing, Information Computing and Communication (ICGTSPICC), 2016, pp. $298-301$. 